\title{
A Particular Case of Pubic Pain
}

STEFANO STAGI, MD, Health Sciences Department, University of Florence, Pediatric Endocrinology Unit, Anna Meyer Children's University Hospital, Florence; SAMUELE G. GRAGNANI, MD; FABRIZIO MICHELOTTI, MD, Pediatric Unit, Cecina Hospital, Livorno; MAURIZIO de MARTINO, MD, $\mathrm{PhD}$, Health Sciences Department, University of Florence, Anna Meyer Children's University Hospital, Florence, Italy. Address correspondence to Dr. Stefano Stagi, Health Sciences Department, University of Florence, Pediatric Endocrinology Unit, Anna Meyer Children's University Hospital, viale Pieraccini 24, 50139, Florence, Italy. E-mail: stefano.stagi@yahoo.it. J Rheumatol 2014;41:2487-9; doi:10.3899/jrheum.140090

The enlargement of the ischiopubic synchondrosis is a frequent occurrence in healthy children, and often disappears after skeletal maturation, termed ischiopubic osteochondrosis ${ }^{1}$.

A 9.6-year-old, right-footed white boy presented right pubic pain for 3 months. He reported that the pain was more pronounced while engaging in sports activities. No history of current or prior injury or infection was reported.

At the time of evaluation, the child was apyretic with pronounced tenderness over the descending ramus of the right pubis, without swelling or redness. Laboratory tests revealed a slight increase in the C-reactive protein level and erythrocyte sedimentation rate. The blood count revealed polymorphonuclear leukocytosis. Other laboratory tests were unremarkable.

Radiographs revealed a rarefaction area with roughening and thickening at the right ischiopubic junction (Figure 1A). A magnetic resonance imaging (MRI) examination (short-tau inversion recovery and fat-saturation images) confirmed a circumscribed deformity identified as ischiopubic synchondrosis with $\mathrm{T} 2$ signal hyperintensity of the bone marrow of the right ischiopubic ramus (Figure 1B).

The diagnosis was ischiopubic osteochondrosis, a poorly understood disorder, and the patient was treated with rest. After 5 months, the child was asymptomatic. Subsequent radiographs revealed complete regeneration of the ischiopubic junction (Figure 2).

Since its first description in $1923^{2}$, enlargement of the ischiopubic synchondrosis has been the subject of controversy because of its uncertain origins ${ }^{3}$. However, because of its frequent occurrence in healthy children and its disappearance after skeletal maturation, the term "ischiopubic osteochondrosis" was introduced ${ }^{1}$. In fact, ossification of the cartilage of the synchondrosis can show variable patterns and velocities, and bilateral fusion is complete in about $80 \%$ of children by the age of 12 years ${ }^{1,4}$. Con- sequently, the appearance of an ischiopubic osteochondrosis could be related to microtrauma acting at the ischiopubic junction, such as other osteochondrosis ${ }^{4}$. During certain athletic activities, such as those involving jumping or kicking, the mechanical forces exerted on the ischiopubic synchondrosis are increased ${ }^{4}$.

However, differences in size and shape of the ischiopubic synchondrosis may make the diagnosis difficult ${ }^{5}$, particularly if it occurs unilaterally and is associated with pain ${ }^{1,4}$. In fact, stress fractures, posttraumatic osteolysis, osteomyelitis, and a neoplastic lesion should be considered as differential diagnoses ${ }^{1,4}$. Many MRI findings may be nonspecific, even if the well-defined margins of the ischiopubic bone support ruling out a neoplastic lesion ${ }^{5}$.

This entity may be common during adolescence, and has a distinct appearance on plain radiographs. Other types of imaging should be avoided because of the possibility of misleading findings and confusion with pathologic lesions. However, pediatricians, rheumatologists, and radiologists should be careful when reporting a suspected lesion in the pubic area of a child during or close to the first decade of life, and should consider ischiopubic osteochondrosis ${ }^{1,4}$.

\section{REFERENCES}

1. Herneth AM. Asymmetric closure of ischiopubic synchondrosis in pediatric patients: correlation with foot dominance. AJR Am J Roentgenol 2004;182:361-5.

2. Delitala F. [Radiological features of coxa-plana]. [Article in Italian] Radiol Med 1923;X:68-9.

3. Canepa G, Stella G. [Juvenile osteochondrosis - Ischiopubic osteochondrosis - Valtancoli-Van Neck disease]. In: Canepa G, Stella G, eds. Textbook of pediatric orthopedics. [Book in Italian.] Padua: Piccin; 2002:1509-10.

4. Ravaglia M. [Delitala-Valtancoli disease: ischio-pubic transitory hypertrophic chondropathy].[Article in Italian] Minerva Med 1967;58:652-4.

5. Oliveira F. Differential diagnosis in painful ischiopubic synchondrosis (IPS): a case report. Iowa Orthop J 2010;30:195-200.

Personal non-commercial use only. The Journal of Rheumatology Copyright @ 2014 . All rights reserved. 
A

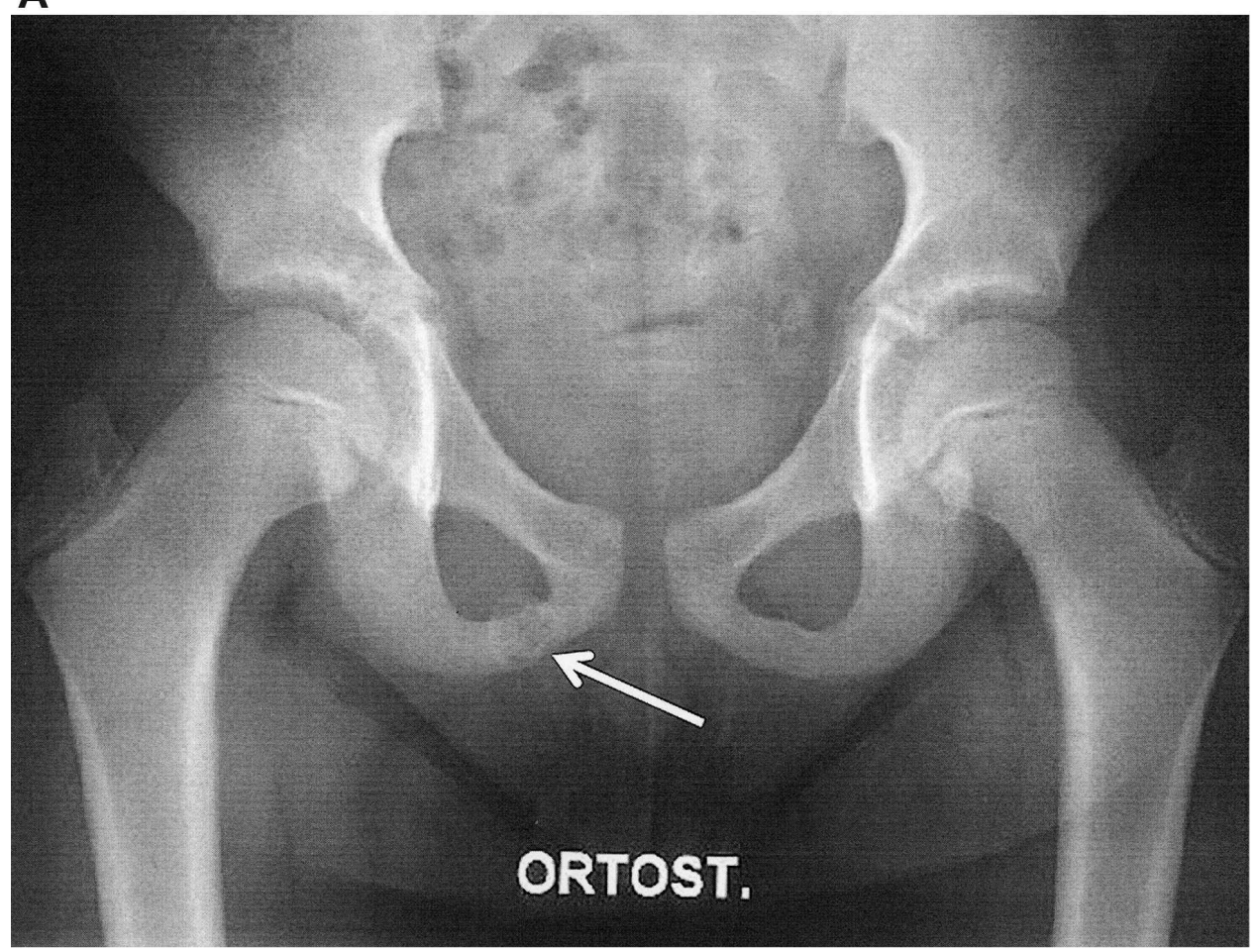

B

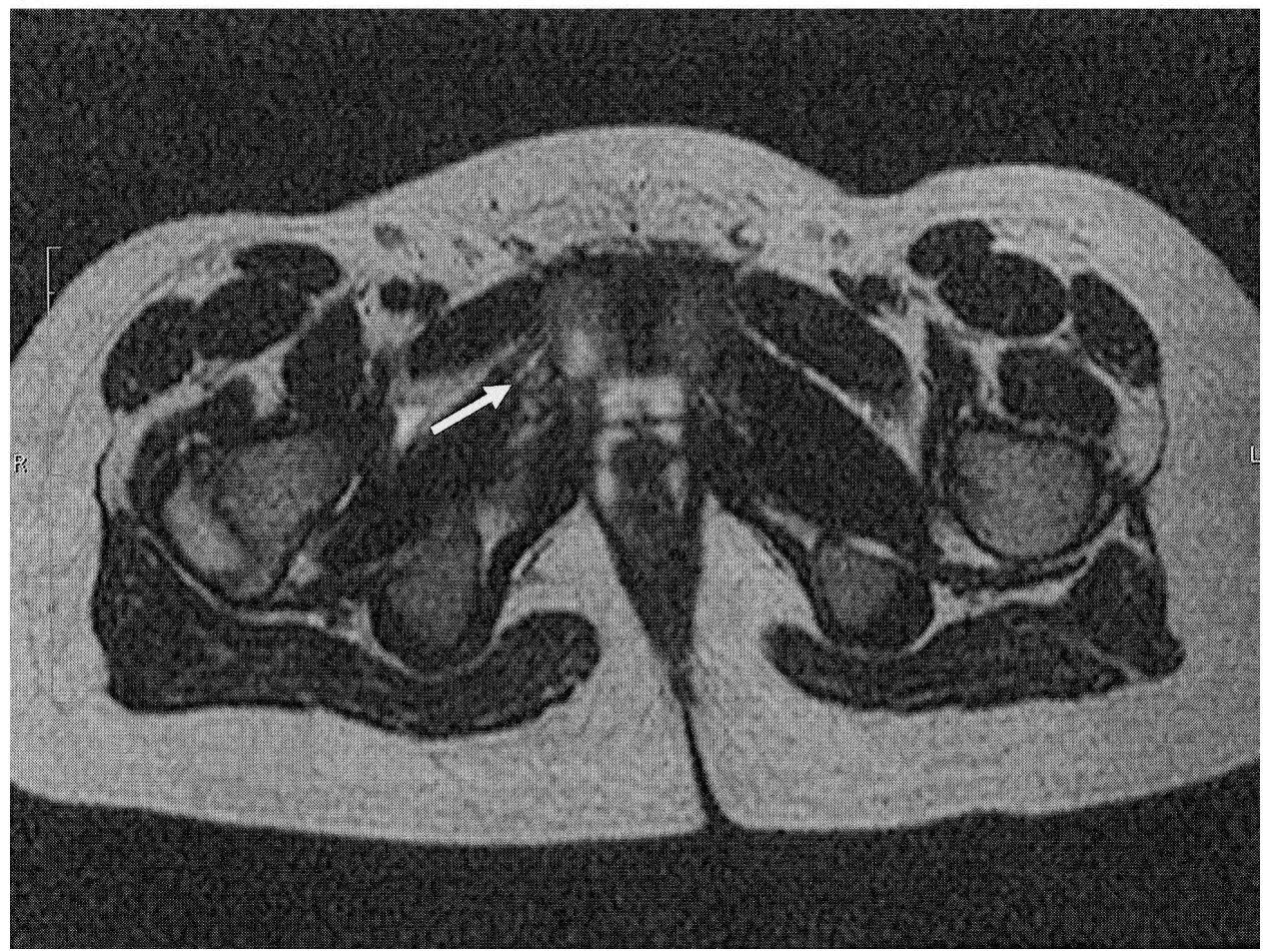

Figure 1. A. Plain film [anteroposterior (AP) view] of the pelvis showing both ischiopubic rami. A view of the right ramus with an enlarged ischiopubic synchondrosis with osteolysis and lucencies (white arrow). B. Plain film (AP view) of the pelvis showing both ischiopubic rami. MRI of the pelvis (axial T2-weighted sequence) showing a hyperintense signal alteration (white arrow) of the right ischiopubic bone marrow and the adjacent soft tissues. MRI: magnetic resonance imaging. 


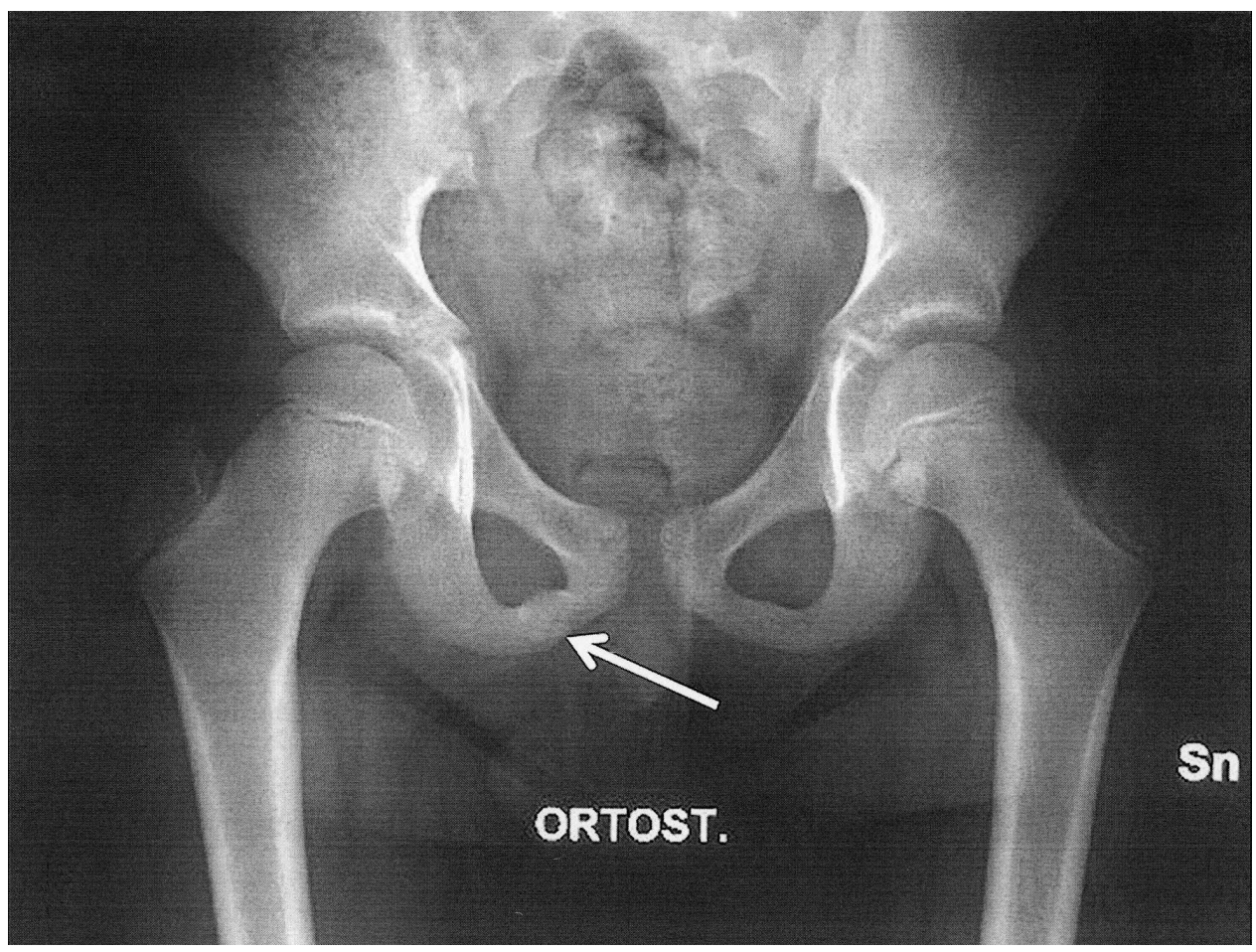

Figure 2. The normalization of the right ischiopubic ramus after 5 months of rest (white arrow). 